\title{
1 State-dependent representations of mixtures by the olfactory bulb
}

Aliya Mari Adefuin*, Sander Lindeman*, Janine Kristin Reinert*, Izumi Fukunaga

Sensory and Behavioural Neuroscience Unit, Okinawa Institute of Science and Technology Graduate

6 University

$7 \quad *$ These authors contributed equally

\section{Abstract}

Sensory systems are often tasked to analyse complex signals from the environment, separating relevant from irrelevant parts. This process of decomposing signals is challenging when a mixture of signals does not equal the sum of its parts, leading to an unpredictable corruption of signal patterns. In olfaction, nonlinear summation is prevalent at various stages of sensory processing. Here, we investigate how the olfactory system deals with binary mixtures of odours under different brain states, using two-photon imaging of olfactory bulb (OB) output neurons. Unlike previous studies using anaesthetised animals, we found that mixture summation is more linear in the early phase of evoked responses in awake, head-fixed mice performing an odour detection task, due to dampened responses. Despite this, and responses being more variable, decoding analyses indicated that the data from behaving mice was well discriminable. Curiously, the time course of decoding accuracy did not correlate strictly with the linearity of summation. Further, a comparison with naïve mice indicated that learning to accurately perform the mixture detection task is not accompanied by more linear mixture summation. Finally, using a simulation, we demonstrate that, while saturating sublinearity tends to degrade the discriminability, the extent of the impairment may depend on other factors, including pattern decorrelation. Altogether, our results demonstrate that the mixture representation in the primary olfactory area is state-dependent, but the analytical perception may not strictly correlate with linearity in summation.

\section{Introduction}

As animals in nature navigate through their environment in order to find food, mates, and to avoid dangers, their sensory systems are tasked to detect and recognise signals of interest despite a background of interfering signals. This figure-ground segregation is a ubiquitous task for many, if not all, sensory systems. In the visual system, for example, segmentation of spatial patterns of light allows animals to recognise objects despite some parts being obscured (Marr, 1982). In the auditory system, spectral combinations of sound waves are recognized and strung together over time to form a stream, allowing animals to recognise social calls from specific individuals among other noises (Bregman, 1990). In olfaction, too, animals face challenges in identifying an odour of interest in the presence of other molecules (Laing and Francis, 1989; Rokni et al., 2014). 
Mármol et al., 2021; Malnic et al., 1999), each odour molecule may activate a number of olfactory receptor types, leading to combinatorial representations (Malnic et al., 1999). As a result, when several compounds are present in a given mixture, they can activate overlapping sets of olfactory receptors, causing complex pharmacological interactions. For example, molecules may bind a common receptor, which, depending on the efficacy, can lead to antagonism (Cruz and Lowe, 2013; Kurahashi et al., 1994; Oka et al.; Reddy et al., 2018; Singh et al., 2019) or enhancement (Xu et al., 2020). Recent large-scale studies demonstrate that this is a widespread phenomenon (Inagaki et al., 2020; Xu et al., 2020; Zak et al., 2020), which means that neural responses to mixtures often do not equal the sums of responses to the individual odours. In addition to the interactions at the periphery, there are many forms of nonlinear summation at multiple stages of olfactory processing, including the saturation of neural responses (Firestein et al., 1993; Wachowiak and Cohen, 2001) and inhibitory interactions in the olfactory bulb (Economo et al., 2016). Widespread suppressive interactions are also observed in downstream areas, including the piriform cortex (Penker et al., 2020; Stettler and Axel, 2009).

Nonlinear summation of signals in some brain areas may be desirable when specific combinations of signals carry special meanings (Agmon-Snir et al., 1998; Jacob et al., 2008). However, in primary sensory areas, it is sometimes considered information limiting (Laughlin, 1989). For olfaction, this is thought to limit the analytical ability - whether a mixture can be perceived in terms of the constituent qualities (Bell et al., 1987; Jinks and Laing, 1999; Laing and Glemarec, 1992). Nonlinear summations, or "interactions", do not occur for all odour mixtures (Fletcher, 2011; Gupta et al., 2015; Tabor et al., 2004), but occur prevalently when the background and target activation patterns overlap. This happens when a mixture contains many components (Mathis et al., 2016), as well as when the background odours are structurally related to the target odour (Cruz and Lowe, 2013; Fletcher, 2011; Jinks and Laing, 1999; Kay et al., 2003; Mathis et al., 2016; Tabor et al., 2004). Nonlinear summation poses a difficulty because it may distort a pattern of interest brought by non-uniform addition of unpredictable background patterns, although more recent studies suggest beneficial effects of antagonism, for example by reducing saturation-related loss of information (Reddy et al., 2018). Due to this difficulty, some studies have suggested that the olfactory system may not decompose mixture representations into component parts, but may solve the task by learning task-specific boundaries instead (Mathis et al., 2016; Wilson and Stevenson, 2003).

The question therefore remains: how does the mammalian olfactory system deal with nonlinear summation of responses? To investigate this, we used binary mixtures of odours to investigate mixture representations in mice performing tasks. While temporal structures caused by turbulence may be used to segregate odours of interest from the background (Ackels et al., 2021; Hopfield, 1991), we tackle the case when temporal information from the environment is not available. We demonstrate, by comparing the mixture responses using various paradigms, that the property of mixture summation depends on the brain state. 


\section{Results}

Olfactory figure-ground segregation is most difficult when the target and background odours evoke overlapping activity patterns (Rokni et al., 2014). To study this task using binary mixtures, we first characterized how our target odour relates to other odours in our panel. The target odour was ethyl butyrate and the rest of the odours in our set comprised a range of small esters structurally similar to ethyl butyrate, as well as non-esters (Fig. 1A). According to Rokni et al., the masking index, which measures the amount of overlap in response patterns between the target odour and background odours, correlates well with behavioural performances (Rokni et al., 2014).

To measure the masking indices from single odour responses, we obtained activity patterns from the $O B$ output neurons, the mitral and tufted (M/T) cells. Using a two-photon microscope, we imaged from the glomerular layer of the olfactory bulb in Tbet-Cre::Ai95D mice, which express the calcium indicator GCaMP6f (Dana et al., 2019) in M/T cells (Haddad et al., 2013). To reproduce previous results, these initial imaging experiments took place in mice under anaesthesia (Fig. 1B). We studied the degree of overlap between ethyl butyrate and other odour responses by presenting single odours in a randomized order. An analysis of glomerular activity patterns revealed that methyl butyrate responses overlap the most with the ethyl butyrate response patterns, followed by closely related esters (Fig. 1C-E).

Previous studies from anaesthetised animals showed that neural responses to odour mixtures exhibit widespread nonlinear summation (Inagaki et al., 2020; Oka et al.; Reddy et al., 2018; Singh et al., 2019; Xu et al., 2020). In particular, suppressive interactions become dominant among large responses, due to the saturation effect (Mathis et al., 2016). This pattern is observed at many stages of olfactory processing, including in the olfactory bulb (Economo et al., 2016; Fletcher, 2011) and anterior piriform cortex (Penker et al., 2020), but it depends on the complexity of mixtures, as well as odorant choices (Fletcher, 2011; Gupta et al., 2015; Rokni and Murthy, 2014; Tabor et al., 2004). We therefore characterized the property of binary mixture summation using our odour set.

We first confirmed that our olfactometer is capable of presenting stable concentrations of odours for mixtures. We used a photoionisation detector to ensure that, when two odours are mixed, ionisation levels sum linearly (Fig. $2 A-C$ ). Then, to assess how the $O B$ output represents binary mixtures, we imaged the individual somata of $\mathrm{M} / \mathrm{T}$ cells in Tbet-cre::Ai95D mice (Fig. 2D). A typical session consisted of about 40 trials, to minimise time-dependent effects. Single odours and their binary combinations were presented in a semi-random order. Since ethyl butyrate, methyl butyrate, and the mixture of these two odours are of particular importance in this study, these three trial types appeared every 10 trials (Fig. 2E; see methods). To assess how the mixture of ethyl butyrate and methyl butyrate is represented, the amplitudes of the mixture responses were compared against those of linear sums of single odour responses (Fig. 2F,G). As reported before, a large proportion of $M / T$ cells exhibited nonlinear summation (39.0\% of $\mathrm{M} / \mathrm{T}$ cells showed a deviation from linearity greater than $2 ; 71 / 182$ ROIs, 7 fields of view, 4 mice; see methods) with a large fraction showing sublinear summation $(35.7 \%$, $65 / 182$ ROIs).

Given that nonlinear summation is so widespread in the $\mathrm{OB}$ output, how well can mice analyse binary mixtures to accurately detect the presence of the target odour at the behavioural level? To assess this, 
we used a Go/No-Go paradigm for head-fixed mice. The rewarded stimulus (S+ odour) contained ethyl butyrate, either as a single odour or as a component of binary mixtures (Fig $3 \mathrm{~A}$ ). To train mice on this task efficiently, after habituation, the head-fixed mice were first trained to discriminate ethyl butyrate against other single odours (Fig 3A). The mice learned to perform this task well, reaching an accuracy of $80 \%$ within 200 trials on average (Fig. 3 B,C; number of trials to reach $80 \%$ accuracy $=194.3 \pm 21.9$; $n=7$ mice). Subsequently, these mice were trained to detect the presence of ethyl butyrate in binary mixtures (Fig. 3B,C). The mice performed the mixture task at a high accuracy from the beginning $(90.2$ $\pm 1.3 \%$ of trials with correct responses in the first session; $n=7$ mice). However, the mistakes they made were odour-specific, in that they tended to lick more indiscriminately on methyl butyratecontaining trials regardless of the presence of ethyl butyrate (Fig 3D,E; mean lick preference index for $\mathrm{MB}$ trials $=0.74 \pm 0.04$ vs. 0.93 for 5 other background odours, $p=0.0035$, 1-way ANOVA; $n=7$ mice). However, with training, the performance on methyl butyrate trials, too, became accurate, demonstrating that mice can learn to accurately detect the target odour in binary mixtures even when the background odour is similar.

To understand why mice were able to acquire the binary mixture task so easily, we analysed how M/T cells represent binary olfactory mixtures while the trained mice accurately performed the task. GCaMP6f fluorescence was measured from M/T cell somata. Data from the behaving mice was compared to the case when the same mice were later anaesthetised with ketamine and xylazine (Fig $4 A-D)$. This revealed that the $M / T$ cells in behaving mice tended to sum mixture responses more linearly than when the mice were anaesthetised (Fig. 4D). To quantify this data, we expressed a deviation from the linear sum as a fraction of the predicted, linear sum of component responses ("fractional deviation"; Fig. 4E). Data from awake, behaving mice showed a clear shift in the distribution of this index, compared to the anaesthetised case. In the anaesthetised case, a steep slope in the cumulative histogram occurs at a negative value (median fractional deviation $=-0.39$ ), indicating that the majority of the $\mathrm{OB}$ output neurons exhibit sublinear summation. On the other hand, $\mathrm{M} / \mathrm{T}$ cells from behaving mice showed a broader distribution centred around 0 (median fractional deviation = $0.12 ; p=3.83 \times 10^{-10}$, two-sample Komogorov-Smirnov test comparing the behaving vs. anaesthetised data; $\mathrm{n}=202 \mathrm{ROIs}$ and $103 \mathrm{ROIs}$, respectively). The broader distribution for the awake, behaving condition likely reflects a greater variability in this condition. Consistent with this, adding a Gaussian noise to the data from anaesthetised mice made the distribution broader, but without affecting the median (Fig. 4F). Using this index to compare the two conditions, we found that the difference was most striking in the first second after the odour onset ("early phase"; Fig. 4G). Further, we observed that the responses in the somata are more linear at an early phase compared to the apical dendrites (Fig. 4 -supplement 1). These observations together may indicate that dampened responses in the early phase of somatic responses may underlie the reduced mixture suppression, although some contribution may come from more decorrelated responses at the somata (Fig. 4 - supplement 1 ). Overall, the results indicate that the property of mixture summation is more linear in the awake, behaving mice, and that linearisation is not imprinted permanently as a result of learning.

While the above result demonstrates that mixtures sum more linearly in awake, behaving mice, M/TC responses are also significantly smaller and more variable in this condition. This is quantified in an analysis of trial-by-trial response similarity, which measures the Pearson correlation coefficient between population responses from individual trials (Fig. 5A-C). This showed that, in the anaesthetised mice, the $\mathrm{M} / \mathrm{TC}$ response patterns are highly correlated, both within class (comparison 
between trials with ethyl butyrate) and across classes (Fig. 5A,B). In the awake, behaving mice, as expected, responses were less consistent, indicated by the lower within-class correlation (Fig. 5A,B). However, because across-class patterns were substantially less correlated (Fig. 5A,B), S+ responses may be more discriminable in the behaving mice.

The ability to reliably encode the presence of ethyl butyrate was first quantified based on within-class vs. across-class correlation, by counting the proportion of trials where within-class correlation was higher than across-class (Bridgeford et al., 2021). This indicated that $M / T$ cells from anaesthetised mice showed better discriminability initially, but later, $M / T$ cells in the behaving mice performed as well as the anaesthetised mice (Fig. 5C). Note a similar result is obtained when trials are sorted by the presence of methyl butyrate (Fig. 5 supplement 1). While useful, the Pearson correlation coefficient takes into account all ROls, irrespective of how informative they are in discriminating patterns, and may not reflect the true ability of a population of neurons to encode odours. To address this, we trained support vector machines (SVMs) to discriminate responses evoked by odours containing ethyl butyrate (S+) vs. odours without ethyl butyrate (S-). We used $80 \%$ of randomly selected trials from each session for training, and the remaining $20 \%$ of the trials to test the performance (Fig. 5D). This time, $\mathrm{M} / \mathrm{T}$ cells from the two conditions performed similarly for the first 1 second, performing above chance soon after the odour onset (earliest time where accuracy is significantly above $0.5=0.67 \mathrm{~s}$ for the behaving case, and $1.12 \mathrm{~s}$ for anaesthetised data; t-test at significance level of $0.05 ; n=13$ fields of view, 6 mice for behaving case; 8 fields of view, 4 mice for the anaesthetised case; Fig. 5D). Further, the data from behaving mice outperformed the anaesthetised case in the later phase (median accuracy for 2-3 s after odour onset $=0.58$ vs. 0.70 for anaesthetised vs. behaving mice, respectively; $p=0.027$, Mann-Whitney $U$ test for equal medians). We also wished to assess how mixture responses relate to single odour patterns. To do so, we trained SVMs using single odour responses (EB vs. other single odours) and tested the performance on mixture responses (Fig. 5E). Again, the data from behaving mice outperformed the data from anaesthetised mice in the late phase although the difference was not statistically significant (median accuracy for 2-3 s after odour onset $=0.55$ vs. 0.64 for anaesthetised vs. behaving mice, respectively; $p=0.065$, Mann-Whitney $U$ test for equal medians). This may suggest that, over time, mixture responses come to resemble the target component odour pattern more in the awake, behaving mice than in the anaesthetised mice. Curiously, the time course of decoder accuracy did not strictly depend on the linearity of mixture summation (Fig. 6 - figure supplement 2). Overall, the result suggests that, despite the substantial differences in the amplitudes and variability of responses, $\mathrm{M} / \mathrm{T}$ cells in behaving mice are able to encode the presence of the target odour well.

To what extent is the mixture representation in the olfactory bulb affected by learning? Previous studies suggest that prior exposures and familiarity to odours affect the ability to analyse odour mixtures (Grabska-Barwińska et al., 2017; Poupon et al., 2018). To address this, we assessed how ethyl butyrate and methyl butyrate mixtures are represented in naïve but awake, head-fixed mice. Since different levels of motivation, with accompanying changes in the sniff patterns, affect the olfactory system (Carey and Wachowiak, 2011; Jordan et al., 2018), we made one group naïve mice engaged and a second group of mice disengaged, by changing the reward contingency (Fig. 6A). That is, we presented the same sets of odour stimuli, but instead of associating specific odours with reward, the water reward was either delivered after odour onset but on randomly selected trials to engaged the mice, or about 15 seconds before the odour onset on all trials in order to disengage the mice (Fig. 6A; 
$\mathrm{n}=17$ sessions, 6 mice and 14 sessions, 4 mice). The level of engagement was confirmed by the speed of inhalations during odour presentations (Fig. 6B) and the number of anticipatory licks generated (Fig. 6 - figure supplement 1 ).

In all awake conditions, we found the ethyl butyrate and methyl butyrate responses to sum equally linearly (Fig. 6 - figure supplement 1), indicating that the linearity of summation does not depend on the behavioural states or learning. However, the performance of SVMs on these datasets showed a state-dependence. While the general ability to discriminate S+ vs. S- odours was comparable across the states, the ability to analyse mixtures in terms of the constituents - SVMs trained on single odours and tested on mixture patterns - was particularly poor for the disengaged mice (Fig. 6C-F). Since the linearity of summation was comparable across the three groups (Fig.6 - figure supplement 1), this, again, suggests that the ability to correctly decode the presence of odour does not strictly correlate with the linearity of mixture summation. In all cases, the decoder performed most accurately in the late phase. We hypothesized that this extra time may reflect an involvement of recurrent interactions with the piriform cortex, which is thought to store learned representations and feed back to the olfactory bulb (Chapuis and Wilson, 2012; Grabska-Barwińska et al., 2017). Our preliminary result from muscimol infusion in the ipsilateral piriform cortex, however, suggests that this may not be the case (Fig. 6 supplement 3).

Under what circumstance does discriminability of odour mixtures decouple from linearity in mixture summation? To study the effect of saturating sublinearity in isolation, we made a simulation as follows. Linear sums of responses were constructed by adding component responses obtained from the imaging sessions. In one test, these sums, with added noise, were passed through SVMs that had been trained with component responses. In another test, the linearly summed responses with noise were transformed with a normalising function (Mathis et al., 2016; Penker et al., 2020), which adds sublinearity in an amplitude-dependent manner (Fig. 7B; see methods). Then, these signals were passed through the same SVMs to assess how discriminable the activity patterns were. When the data from the anaesthetised mice was used, normalising sublinearity was particularly detrimental around 2 seconds after the odour onset (Fig. 7C), qualitatively reproducing the transient decrease in the accuracy seen with the observed mixture responses (Fig. 5E). In contrast, with the data from the behaving mice, normalising sublinearity did not have as significant an effect on the mixture discriminability, even at the later stage when sublinear summation becomes more widespread (Fig. 7D,E). Thus, the functional consequence of nonlinear summation may depend on specific circumstances, for example on how separable, or decorrelated, the activity patterns are. Overall, our result indicates that mixture responses in the olfactory bulb are highly state-dependent and evolve over time (Fig. 6F).

\section{Discussion}

Segmentation and extraction of relevant information from mixtures of signals are important and perpetual tasks for sensory systems. Nonlinear summation of signals is generally thought to pose difficulty in demixing component signals. Here, we demonstrate that the signal summation in the primary olfactory area of the mouse is significantly more linear in awake mice. This is notable, because many previous studies reported that suppressive mixture interactions are widespread in the central 
nervous system, based on anaesthetised preparations (Bell et al., 1987; Cruz and Lowe, 2013; Penker et al., 2020; Stettler and Axel, 2009).

However, our analysis also indicates that the ability to discriminate binary mixture responses does not correlate strictly with linearity in summation. First, the behavioural improvement was not accompanied by more linear summation. Second, the time course of decoder accuracy does not follow the linearity of summation. Indeed, in the data from behaving mice, the decoder performance peaked when mixture responses were most sublinear. This raises a question: is sublinear summation in mixtures detrimental, or beneficial to discriminating mixtures of odours? Our simple simulation indicates that, generally, a sublinear mixture summation due to saturating influences limits the discriminability of mixture responses. Thus, dampened responses in awake animals bring some advantage by maintaining the mixture responses in the linear range. However, in the behaving mice, even when the responses became larger at a later phase, the saturating influence was less detrimental to discrimination. Here, decorrelated response patterns may play a more crucial role, as it is known to enhance classifier or behavioural performances (Bhattacharjee et al., 2019; Friedrich and Wiechert, 2014; Gschwend et al., 2015; Padmanabhan and Urban, 2010). While the behavioural task described in this study is simple and animals make accurate decisions within $1 \mathrm{~s}$ (771 $\pm 97 \mathrm{~ms}$ ) of stimulus onset, the slower mechanisms described here may be important when larger and more reliable responses are required for accurate decisions. So, in addition to sampling time (Rinberg et al., 2006), this phenomenon may be a part of mechanisms needed to solve more difficult tasks accurately (Abraham et al., 2010; Wilson et al., 2017).

Mechanistically, the ability to accurately analyse, or discriminate between, mixtures based on the knowledge of component is hypothesised to involve the piriform cortex, through learned representations (Chapuis and Wilson, 2012; Grabska-Barwińska et al., 2017). For tufted cells, however, the relevant sources of feedback could be other brain regions, such as the anterior olfactory nucleus (Chae et al., 2021). It will be an intriguing future investigation to identify the source and mechanisms of state-dependent mixture representations.

Perception of olfactory mixtures has long fascinated investigators. Mixtures of odours often have qualities that are different from those of the individual components. Accurate recognition of components is particularly hard for human subjects. For untrained subjects, olfactory mixtures that contain about 30 components tend to smell alike (Weiss et al., 2012). Even highly trained people like perfumers can only accurately identify individual components if unfamiliar mixtures contained no more than 5 components (Poupon et al., 2018). These demonstrate an ultimate limit in the analytical perception of olfactory mixtures. In addition, in non-expert humans and rodents alike, when the task is to look for a particular aroma in the mixture, the tendency is to falsely report the presence of the target odour in target-odour detection tasks (Laing and Glemarec, 1992; Rokni et al., 2014). In all cases, an extensive training for specific odours can improve the ability to detect the target odour, as seen in the case for sommeliers who routinely analyse key components in wines, which can contain severalhundred component mixtures (Ilc et al., 2016). Thus, while mixture perception is highly context specific (Rokni and Murthy, 2014), training in specific odours seems to be key to improving on mixture analysis. With more complex mixtures, mechanisms within the olfactory bulb may reach a limit, and also is unlikely to be the only mechanism that is used to solve the task. Elucidating what roles the 
bioRxiv preprint doi: https://doi.org/10.1101/2021.09.23.461425; this version posted February $22,2022$. The copyright holder for this

preprint (which was not certified by peer review) is the author/funder, who has granted bioRxiv a license to display the preprint in perpetuity. It is made available under aCC-BY-ND 4.0 International license.

primary sensory areas plays is a crucial step towards a mechanistic understanding of complex sensory processing.

\section{Acknowledgement}

We would like to thank Yu-Pei Huang and OIST's Animal Resource Service staff for their dedicated assistance, the members of Andreas Schaefer's group and Kevin Franks' group for comments on earlier versions of the work, and Adam Mago, Xiaochen Fu, and Josefine Reuschenbach for comments on the manuscript. This work was supported by the OIST Graduate University. 
Methods

Animals

All animal experiments have been approved by the OIST Graduate University's Animal Care and Use Committee (Protocol 2016-151 and 2020-310). Thet-Cre (Haddad et al., 2013) and B6J.Cg-

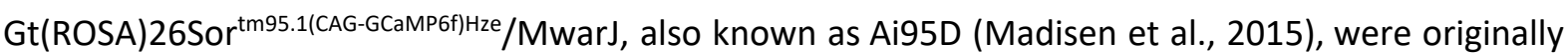
obtained from Jackson Laboratory (stock numbers 024507 and 028865, respectively). Tbet-Cre::Ai95D mice were generated by crossing homozygous Tbet-Cre and Ai95D mice resulting in heterozygous animals used for imaging experiments. C57BI6J mice were purchased from Japan CLEA (Shizuoka, Japan) and were acclimatized to the OIST facility for at least 1 week before they were used for experiments. All mice used in this study were adult male (8-11 weeks old at the time of surgery).

\section{Olfactometry}

A custom-made flow-dilution olfactometer was used to present odours. Briefly, custom Labview codes were used to control to control solenoid valves, and flow controller (C10054S2-2L-N2, FCON, Japan) was used to regulate the rate of air flow. A pair of normally closed solenoid valves was assigned per odorant and used to odorise the air. These solenoid valves were attached to a manifold, such that a set of 8 pairs had access to the common stream of air. To generate binary mixtures of odours while keeping concentrations stable, one odour from each manifold (lines A \& B) was used. For single odour presentations, odour was mixed with air passed through an empty canister. The odorised air was directed towards the animal only when the solenoid valve closest to the animal (final valve) opened. Final valve was opened for a short time $(0.1 \mathrm{~s}$ for experiments involving behavioural analysis only, and $0.5 \mathrm{~s}$ for all imaging experiments, to accommodate for slow respirations during anaesthesia), to avoid adaptation related to temporal filtering for high sniff frequencies associated with long odour pulses (Verhagen et al., 2007). Odours were presented at 1$5 \%$ of the saturated vapour. Total air flow, which is a sum of odorised air and the dilution air, was approximately $2 \mathrm{~L} / \mathrm{min}$, which was matched by the air that normally flows towards the animal. Intertrial interval was approximately 40 seconds to ensure that the flow controllers have stabilised before each odour presentation. All odorants were from Tokyo Chemical Industry (Tokyo, Japan), apart for ethyl butyrate (W242705), which was from Sigma-Aldrich. Product number were: T0247 (Ethyl tiglate), V0005 (Methyl valerate), A0061 (Acetophenone), A0500 (Methyl anthranilate), B0757 (Butyl butyrate), B0763 (Methyl butyrate), S0015 (Methyl salicylate), S0004 (Salicylaldehyde), T0248 (Methyl tiglate), A0232 (Eugenol). Purity of all odorants was at least $98 \%$ at the time of purchase. Stock odorants were stored at room temperature in a cabinet filled with $\mathrm{N}_{2}$ and away from light.

Head plate implantation: All recovery surgery was conducted in an aseptic condition. 8-11week old male C57BI6/J mice were deeply anaesthetised with isoflurane. The body temprature was kept at $36.5^{\circ} \mathrm{C}$ using a heating blanket with a DC controller (FHC, Bowdoin, USA). To attach a custom head plate about $1 \mathrm{~cm}$ in width weighing a few grams, the skin over the parietal bones was excised and the soft tissue underneath was cleaned, exposing the skull. The exposed skull was gently scarred with a dental drill, cleaned, dried, and coated with cyanoacrylate (Histoacryl, B.Braun, Hessen, Germany) before placing the headplate and fixing with dental cement (Kulzer, Hanau, Germany). For optical window implantation, adult, male Tbet-Cre::Ai95D mice were deeply anaesthetised, and underwent a 
the frontal bone, a craniotomy about $1 \mathrm{~mm}$ in diameter was made over the left olfactory bulb and on the exposed dorsal surface, a cut piece of coverslip that snugly fit in the craniotomy, on the edge of the drilled bone, was gently pressed down and sealed with a cyanoacrylate and fixed with dental cement. Mice were recovered in a warm chamber, returned to their cages, and given carprofen subcutaneously $(5 \mathrm{mg} / \mathrm{kg}$ ) for 3 consecutive days.

\section{Habituation and behavioural measurements}

Water restriction began two weeks after surgery. Mice went through 3 days of habituation to head fixation, one session per day for approximately 30 minutes, until mice learned to lick vigorously for water reward. Respiration pattern was measured by sensing the air flow just outside the right nostril by placing a flow sensor (AWM3100V, Honeywell, North Carolina, USA), and the data was acquired at $1 \mathrm{kHz}$. Lick responses were measured using an IR beam sensor (PM-F25, Panasonic, Osaka, Japan) that was part of the water port. Nasal flow, an analog signal indicating the odours used, lick signal, a copy of the final valve and water valve timing were acquired using a data acquisition interface (Power1401, CED, Cambridge, UK).

\section{Discrimination training}

After habituation, the head-fixed mice were trained to associate a water reward with a target odour (ethyl butyrate). The reward was two droplets of water ( $10 \mu \mathrm{l}$ each), that arrived 3 seconds after the onset of the final valve opening. The mice underwent single odour discrimination training first, until they generated anticipatory licks in response to ethyl butyrate presentations, and correctly refrained from licking in response to other single odours. Once the overall accuracy was above $80 \%$ in at least one behavioural session, the mice went through the mixture detection task. A typical training session comprised roughly 100 trials, lasting about 1 hour. Rewarded trials comprised a third of all trials. Twophoton imaging took place once the mice performed at $80 \%$ accuracy or above.

\section{Random association paradigm}

After habituation, the head-fixed mice were presented with the same odour mixture stimuli as those that underwent the discrimination training. The water reward was delivered on randomly selected trials, 3 seconds after the onset of the final valve opening. One behavioural session was used to accustom the mice to the odours. Two-photon imaging commenced from the second behavioural session.

\section{Disengagement paradigm}

After habituation, the head-fixed mice were presented with the same odour mixture stimuli as above. The water reward was delivered every trial, 15 seconds before the onset of the final valve opening, arriving in the middle of the inter-trial interval, which was 40 seconds to ensure thorough purging to clear the lines, as well as to stabilize the flow controllers. Two-photon imaging commenced from the second behavioural session.

\section{Odour mixture trial composition}

Binary mixtures have been chosen due to the smaller number of possible odour combinations compared to more complex mixtures. However, even with 11 odours, there is a limit in the number of trials each head-fixed mouse can sample in a given session. We therefore decided to focus on the EB $+\mathrm{MB}$ mixtures. However, it was crucial that, $\mathrm{EB}, \mathrm{MB}$, and $\mathrm{EB}+\mathrm{MB}$ mixture are not presented too 
frequently, to avoid possible adaptation, as well as mice becoming over familiar, especially in the EBdetection task, where the goal was not to train mice to remember specific odour combinations. Thus, we used a compromise paradigm where $\mathrm{EB}, \mathrm{MB}$, and $\mathrm{EB}+\mathrm{MB}$ appeared every 10 trials.

\section{Data analysis}

The data was analysed offline using custom Matlab codes. To calculate the accuracy, the number of licks during 3 seconds from the final valve onset was measured for each trial. Threshold for an anticipatory lick was set to 2 , thus the correct response for rewarded trials was 2 or more beam breaks, and the correct response for unrewarded trials was 1 lick or less during the response time window. To calculate the learning curve, the accuracy was expressed as the proportion of correct trials in a given block of 50 trials. Time to inhalation peak: to calculate the speed of inhalation, onset of inhalation and peak of inhalation was detected using Spike2 (CED, Cambridge, UK), using the built-in event detection functions. Briefly, inhalation peaks were detected using the "rising peak" function. These events were used to search backwards in time for the inhalation onset when the flow signal crossed a threshold value. Lick preference index: To measure how well mice discriminated rewarded vs. unrewarded mixtures, anticipatory licking patterns for the two types of trials were compared using the following formula:

$$
\text { Lick preference index }=\left(\text { Lick }_{\text {rewarded }}-\text { Lick }_{\text {unrewarded }}\right) /\left(\text { Lick }_{\text {rewarded }}+\text { Lick }_{\text {unrewarded }}\right)
$$

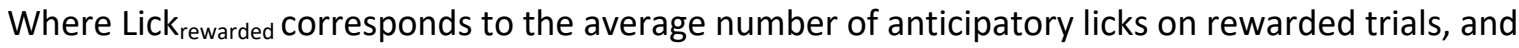
Lick unrewarded corresponds to that for unrewarded trials.

\section{Imaging}

A custom-made two-photon microscope (INSS, UK) with a resonant scanner was used to observe fluorescence from the olfactory bulb of Tbet-Cre::Ai95D mice in vivo. 3D co-ordinates for imaged field of view were recorded relative to the location of a reference, blood vessel pattern on the surface. Unless otherwise stated, imaging from somata was done relatively superficially, just below the glomeruli, thus mainly comprised TCs, which use firing rate modulation to represent odours (Fukunaga et al., 2012). Fields of view for glomerular and somatic levels were $512 \mu \mathrm{m} \times 512 \mu \mathrm{m}$, and $256 \mu \mathrm{m} \times 256 \mu \mathrm{m}$, respectively, and overlapped for awake and anaesthetised conditions, but fewer sessions took place under anaesthesia. In each trial, 400 image frames were acquired at 30 frames per second, with 200 frames before the final valve opening to obtain steady baseline. Unless otherwise stated, the time window analysed for the odour-evoked responses was the first 1 second since the onset of final valve opening. For imaging under ketamine/xylazine anaesthesia (100 mg.kg-1/20 mg. $\mathrm{kg}^{-}$ ${ }^{1}$ intraperitoneally), mice were kept on a warm blanket (FHC, Bowdoin, USA) to maintain the body temperature at $36^{\circ} \mathrm{C}$.

\section{Ipsilateral muscimol infusion in the anterior piriform cortex}

Animals were unilaterally implanted with a 26 -gauge cannula $(10 \mathrm{~mm}$ length; C315GS-4/SPC, Plastics One). Cannula was inserted through a small craniotomy at a stereotactic coordinate [AP, $2.2 \mathrm{~mm}$; ML, $2.4 \mathrm{~mm}$ relative to bregma] and advanced over $6.1 \mathrm{~mm}$ at 45 degree to target the anterior piriform cortex, and fixed with dental cement (Kulzer, Hanau, Germany). For infusion, $500 \mathrm{~nL}$ of $2 \mathrm{mM}$ muscimol (M1523, Sigma-Aldrich, Missouri, USA) was injected using a Hamilton microsyringe, at a rate of 100 $\mathrm{nL} / \mathrm{min}$ approximately 10 minutes before the imaging started. 
Post-hoc verification of infusion cannula placement: Animals were infused with 500nl Dil (Invitrogen, V22885), immediately transcardially perfused with phosphate buffer (225.7 mM NaH $2 \mathrm{PO}_{4}, 774.0 \mathrm{mM}$ $\mathrm{Na}_{2} \mathrm{HPO}_{4}$. pH 7.4) and PFA (4\%, dissolved in phosphate buffer. The heads (including the implants) were postfixed in PFA at $4^{\circ} \mathrm{C}$ for at $48-96$ hours before extraction of the brains. Coronal sections of 100 $\mu \mathrm{m}$ thickness were cut on a vibratome (5100 mz-Plus, Campden Instruments, Leicestershire, UK) and counterstained using DAPI (D9542, Sigma-Aldrich). Images were acquired using a Leica SP8 confocal microscope using a 10X (NA 0.40 Plan-Apochromat, Leica) objective. Images were taken at a resolution of 1024 x 1024 pixels per field of view (1163.64 x $1163.64 \mu \mathrm{m})$.

\section{Image analysis}

Transients were extracted as follows. Regions of interest (ROI) were manually delineated using an average frame from each imaging session. For glomeruli, ROIs were delineated by tracing the outer rim of congregated labelled processes surrounded by a dark region. Pixel values within each ROI were averaged to obtain a time series. Imaging sessions with motion artefacts and drifts were removed from analyses. For each transient, the baseline period was defined as 2 seconds preceding the final valve opening. Relative fluorescence change $(\Delta \mathrm{F} / \mathrm{F})$ was calculated with respect to this baseline. Odour response period was 1 second (30 frames) starting at the onset of the final valve opening, unless otherwise stated. Awake mice tended to adjust sniff patterns, so the final valve opening was not triggered by nasal flow. Masking index for the glomerular level was calculated as previously described (Rokni et al., 2014). Briefly, responses to single odours were obtained from glomeruli of anaesthetised mice. In each field of view, only the glomeruli responsive to the target odour (EB) were analysed. To obtain glomeruli evoked by the target odour, the evoked response amplitudes were converted into zscores. Glomeruli that responded to ethyl butyrate with z-scores higher than 2 were considered. The masking index was the average overlap in the evoked response, where the maximum value for each glomerulus was 1 . For each odour, responses were averaged over 3 or more trials. Mean and standard error of the mean are shown in figures, unless otherwise stated. Boxplots were constructed using the Matlab function boxplot, and show the median, $25^{\text {th }}$ and $75^{\text {th }}$ percentiles, and extreme data points not considered outliers. Outliers are shown with red crosses. The experiments were not done blindly, since the stimulus-reward contingency was visible to the experimenter. However, the olfactometer performance, age and sex of the mice, and analyses codes used were the same for all conditions.

Fractional deviation from linearity was calculated as $\left(R_{\text {observed }}-R_{\text {linear sum }}\right) /\left|R_{\text {linear sum }}\right|$, where $R_{\text {observed }}$ is the mean response amplitude for observed mixture (e.g., response to $E B+M B$ mixture), $R_{\text {linear sum }}$ is the trial average linear sum of component responses (e.g., EB response $+M B$ response. Median fractional deviation was obtained from each field of view. For testing the effect of trial-by-trial variability, Gaussian noise was generated using the Matlab function normrnd with the mean set to 0 , and added to responses from each trial, before the fractional deviation was calculated.

Deviation from linearity was the difference between an observed mixture response amplitude and a linear sum of components normalized by the joint standard deviation: (Robserved $-R_{\text {linear }}$ sum)/(s.e.m.observed + s.e.m.linearsum) 
499 Discriminability index based on correlation coefficient was calculated in the same way as Dicr,

500 described in (Bridgeford et al., 2021), except that the distance measure used was 1 - Pearson's

501 correlation coefficient, instead of the Euclidian distance.

502

503 Decoding analysis: Support vector machines were trained using the Matlab function fitclinear with

504 linear model intercept ("FitBias") set to 0 . Test datasets were used to predict the trial types using the

505 Matlab function predict. Signals were averaged over 30 frames ( 1 second) for each time point to

506 obtain the time course.

507

508 Simulating the effect of normalising sublinearity on discriminability : Support vector machines (SVMs)

509 were trained as above, using the single odour responses only. Then simulated mixture responses were

510 constructed as follows. Single odour responses were averaged over trials. Combinations of two

511 averaged responses were added linearly, and uncorrelated Gaussian noise ( $m e a n=0)$ was added. The

512 noise term comprised two components: fixed, baseline standard deviation ( 0.2 for anaesthetised data,

513 and 2 for awake, behaving case) and standard deviation that scaled with response amplitude, by taking

514 the slope of a linear regression of the observed data. In one test, linear sum with added noise was

515 used as inputs to the above SVMs. In a second set of tests, these simulated responses were further

516 passed through a normalising sublinearity and tested on the same SVMs. The equation of

517 normalization (Penker et al., 2020) was:

$$
R_{j}^{*}=R_{\max }\left(\frac{2}{1+e^{-S \cdot R_{j}}}-1\right)
$$

519 where $R_{j}$ represents the linear-sum amplitude of the $\mathrm{j}^{\text {th }}$ neuron, $R_{j}^{*}$ is the normalized response

520 amplitude of $\mathrm{j}^{\text {th }}$ neuron. Parameters $s$ and $R_{\max }$ were obtained by fitting the data to observed

521 mixture responses (on data from behaving animals at $\mathrm{t}=2$ seconds after odour onset). $R_{\max }$ was 6

522 and $s$ was 0.2 . 
bioRxiv preprint doi: https://doi.org/10.1101/2021.09.23.461425; this version posted February 22,2022 . The copyright holder for this

preprint (which was not certified by peer review) is the author/funder, who has granted bioRxiv a license to display the preprint in perpetuity. It is made available under aCC-BY-ND 4.0 International license.

A
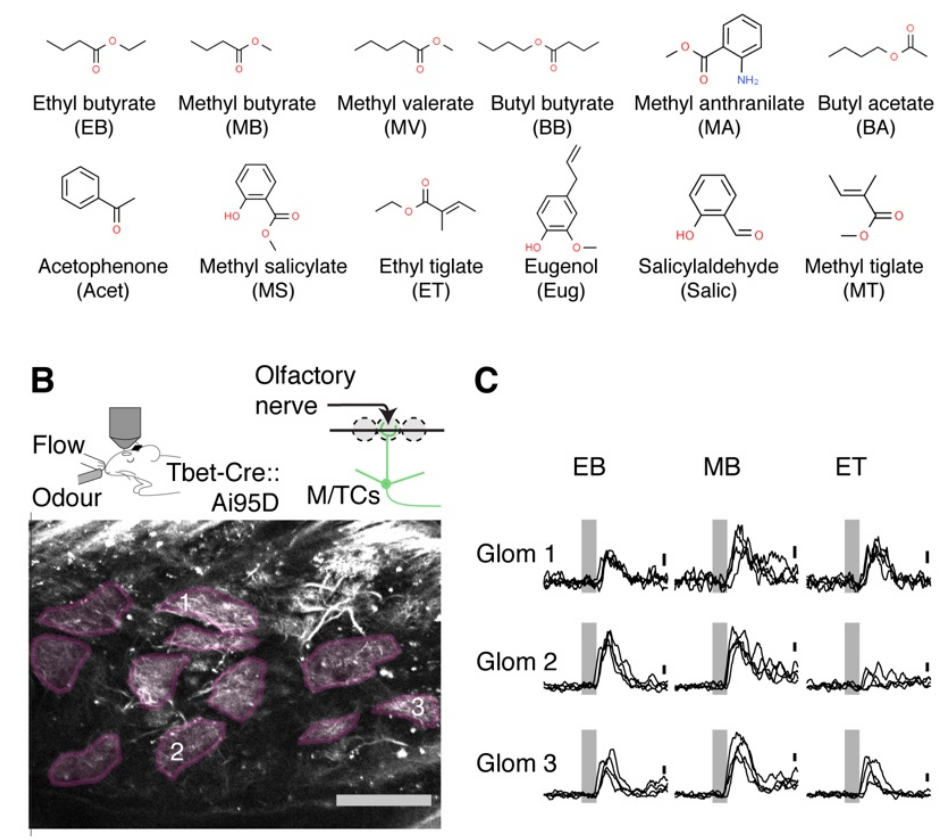

C

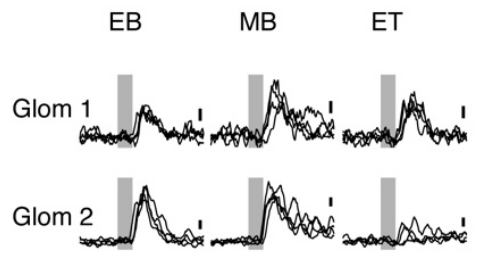

D
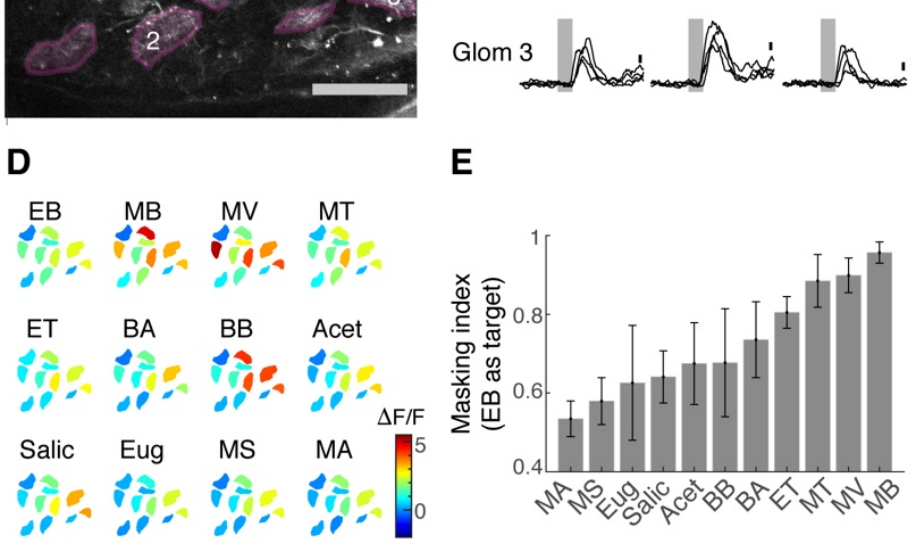

E

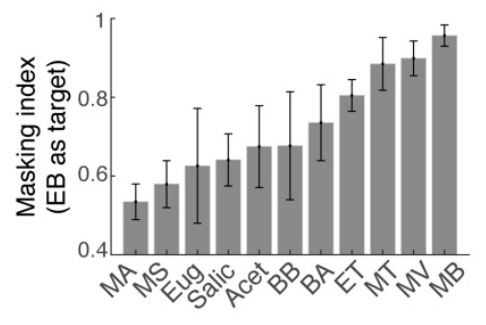

Figure 1: Masking indices of odours used with respect to the ethyl butyrate pattern

(A) Odours in the panel with abbreviations used in the rest of the manuscript. Ethyl butyrate was the target odour for behavioural experiments. (B) Two-photon imaging of GCaMP6f signals from the apical dendrites of $\mathrm{M} / \mathrm{T}$ cells in Tbet-Cre::Ai95D mice under ketamine and xylazine anaesthesia. Scale bar $=0.1 \mathrm{~mm}$. (C) Example GCaMP6f transients expressed as a change in fluorescence $(\Delta F / F)$. Scale bar $=1 \Delta F / F$. Gray $=$ Odour presentation ( $0.5 \mathrm{~s})$. (D) Example of evoked responses. Manually delineated ROIs are shown with fluorescence change evoked by odours, indicated with the corresponding colour map. The amplitude indicated is the average change during 1 second from the final valve opening. (E) Masking indices for all odours in the panel, with EB as the target. $\mathrm{N}=4$ fields of view, 4 mice. Mean and SEM of 3 trials or more shown. 

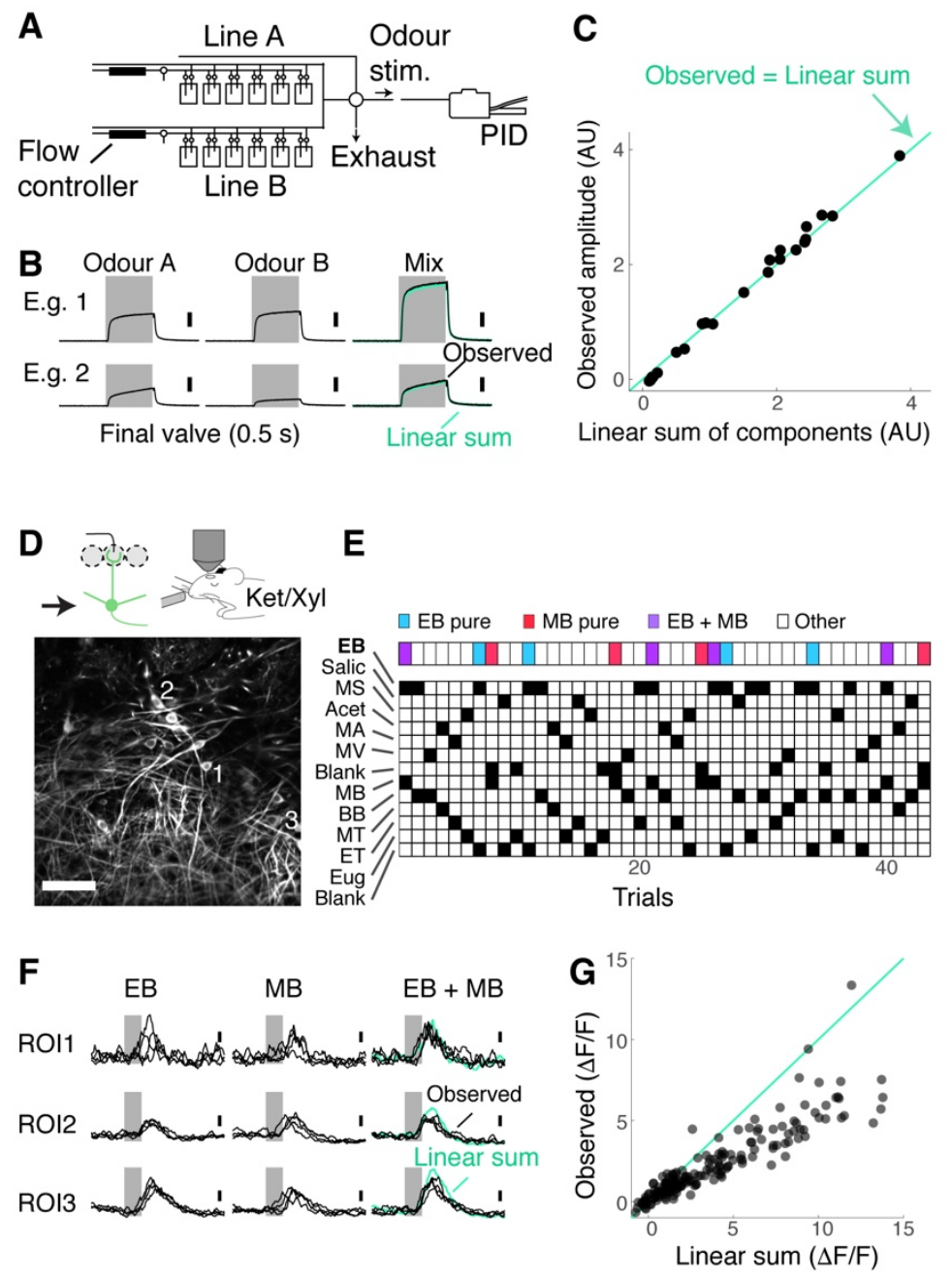

Figure 2: Mixture suppression dominates under anaesthesia

(A-C) Validation of linear mixing by the olfactometer. (A) Binary mixtures are generated by mixing odorized air from two streams each equipped with a mass flow controller and presented as a stimulus when a fiveway valve ("final valve") is actuated. To present single odours, odorized air from one line was mixed with air that passes through a blank canister in the other line. A photoionisation detector (PID) was used for calibration. (B) Example PID measurements for single odours ("Odour A" and "Odour B"), and their mixtures. Linear sum of the components (light blue trace) is shown superimposed with the observed PID signal (black). (C) Observed amplitudes for mixtures vs. linear sum of component amplitudes. Light blue line $=$ the observed mixture amplitude equals the linear sum of components. (D-G) Investigation of mixture summation by M/T cells under anaesthesia. (D) Top: Schematic of two-photon GCaMP6f imaging from somata of M/T cells in naïve Tbet-Cre::Ai95D mice under ketamine/xylazine anaesthesia. Bottom: Example field of view. Scale bar $=50 \mu \mathrm{m}$. (E) Example session structure. Single EB and MB trials, as well as EB+MB mixture trials, are indicated with colour codes. (F) Transients from 3 example ROls as highlighted in $\mathbf{D}$. Linear sum (blue trace) was constructed by linearly summing averages of single EB and MB responses. Gray bar represents time of odour presentation $(0.5 \mathrm{~s})$. (G) Scatter plot of observed mixture response amplitude against linear sum of components. $\mathrm{N}=183$ ROls, 7 fields of view, 4 mice. 
A

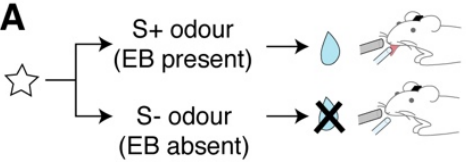

B

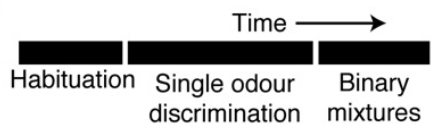

C

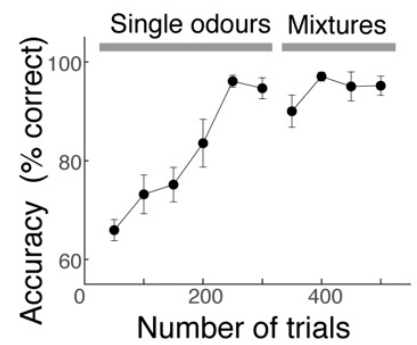

D

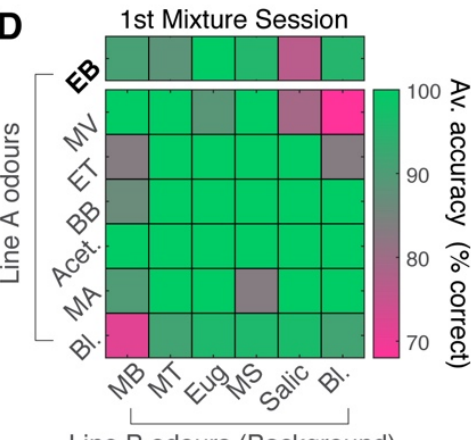

Line B odours (Background)

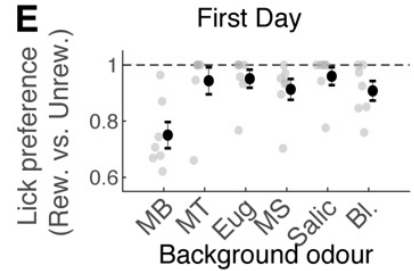

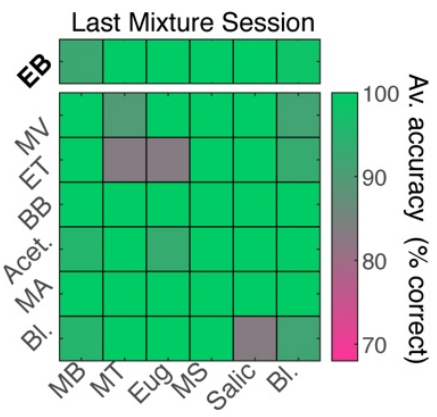

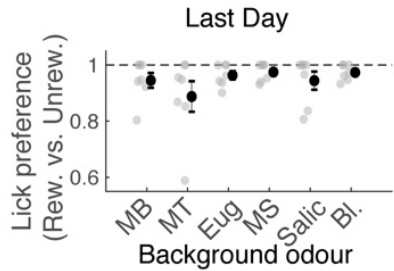

Figure 3: Mice can learn to accurately analyse difficult binary mixtures

(A) Behavioural paradigm: Go/No-Go task with head-fixed mice. EB-containing olfactory stimulus was the rewarded stimulus. (B) After habituation, mice learned to discriminate ethyl butyrate against other single odours in the panel. Once proficient, mice learned to detect the presence of ethyl butyrate in binary mixtures. $30 \%$ of stimuli in the mixture stage were single odours. (C) Behavioural performance for all mice ( $n=7$ mice). Mean and SEM shown. (D) Odour-specific accuracy shown for the $1^{\text {st }}$ (left) and last day of mixture training (right). Green shades indicate high accuracy. Top row corresponds to rewarded trials, and bottom 6 rows correspond to unrewarded trials. Average accuracy from all animals shown ( $n=7$ mice). (E) Lick preference index measures licks that occur preferentially on rewarded trials for a given background odour. A lick preference value of 1 occurs when all anticipatory licks were observed in rewarded trials only. Gray points = data from individual animals ( $\mathrm{n}=7$ mice); Mean and SEM shown in black. 

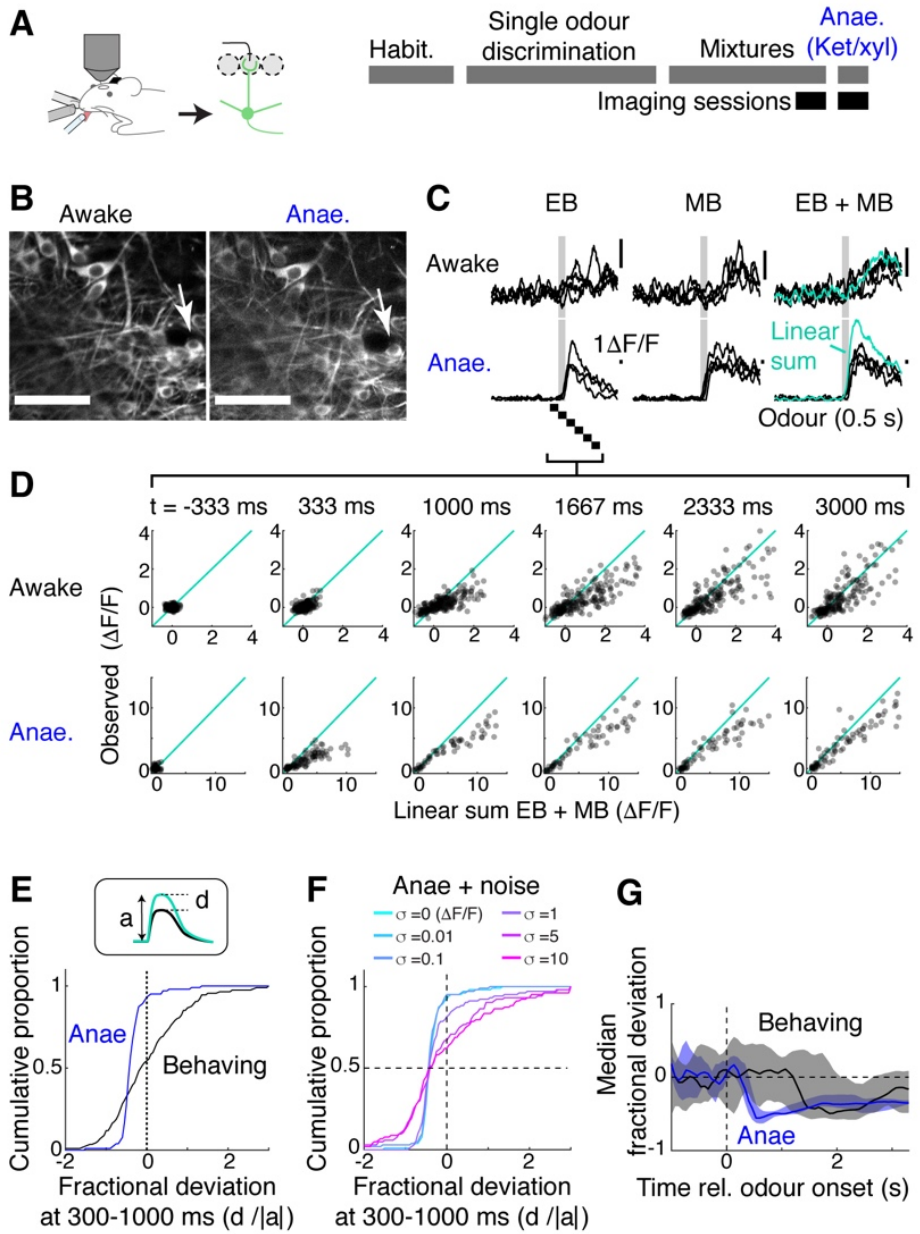

Figure 4: Mixture summation is more linear in awake, behaving mice.

(A) Schematic of experimental setup. Imaging of GCaMP6f signals from the somata in Tbet-Cre::Ai95D mice performing the mixture detection task. On the last day, imaging took place under ketamine/xylazine anaesthesia (Anae). (B) Example field of view showing the same neuron from two imaging sessions. (C) Relative fluorescence change evoked by $\mathrm{EB}, \mathrm{MB}$ and their mixture for the two conditions, for the neuron indicated by arrow in (B). Grey bar = odour presentation (0.5 s). (D) Scatter plot of observed $E B+M B$ mixture response amplitude against linear sum of component odour responses (average of 20 frames). Indicated time is relative to odour onset. (E) Cumulative histograms of fractional deviation from linear sum, for data from anaesthetised mice (blue) and trained mice performing mixture detection task (black). $\mathrm{N}=$ 202 ROIs from 13 fields of view, 6 mice for behaving case; 103 ROIs from 8 imaging sessions, 4 mice for the anaesthetised case. (F) Effect of Gaussian noise on the fractional deviation distribution. Colours correspond to data with different amount of noise added. (G) Time-course of median fractional deviation for the two view, along with $25^{\text {th }}$ and $75^{\text {th }}$ percentiles (shaded areas). See also Figure 4 - figure supplement 1. 
A

$\begin{array}{ll}\text { Examples } & \text { B } \\ \text { Early (t1) Late (t2) Corr. }\end{array}$
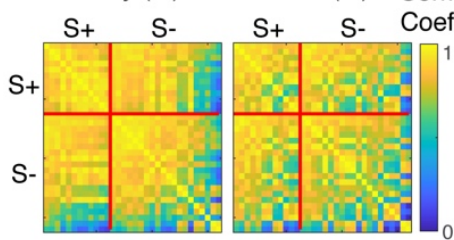

B
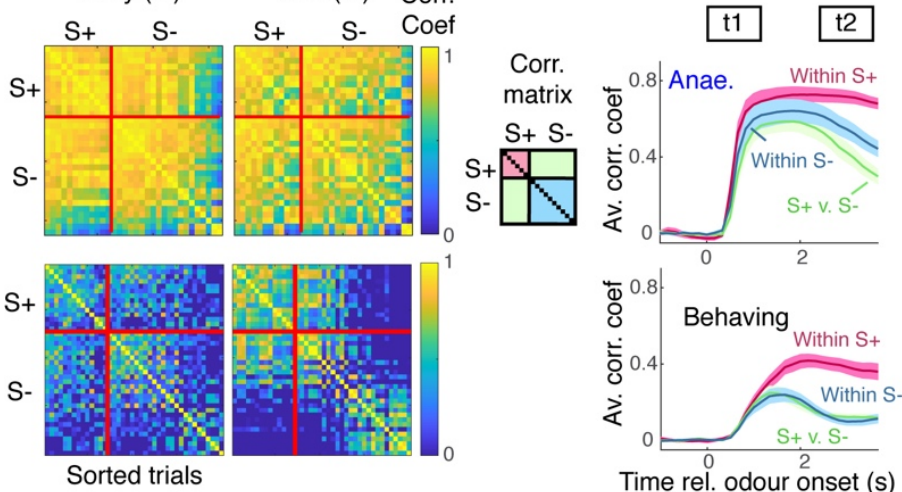

C

D
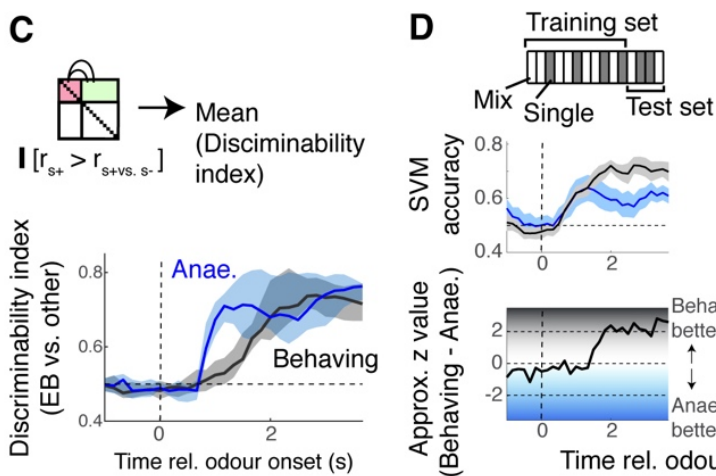

E Training set
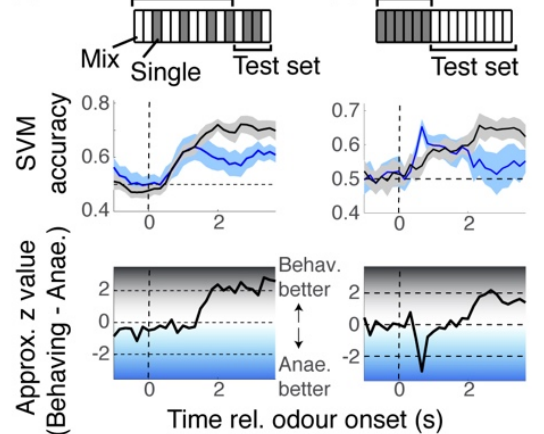

Figure 5: Encoding the presence of the target odour is better in behaving mice.

(A) Correlation matrices for data from anaesthetised mice (top) and awake, behaving mice (bottom), similarity of evoked responses between individual trials, for two time points, $\mathrm{t} 1$ and $\mathrm{t} 2$ as indicated in panel (B). S+ trials were trials with ethyl butyrate either as single odour or in binary mixtures. (B) Average correlation coefficient within S+ trials (magenta), within S- trials (cyan), or across trials (green) for $\mathrm{M} / \mathrm{T}$ cells responses from anaesthetised mice (top panel) and awake, behaving mice (bottom panel). (C) Discriminability index, which measures the proportion of trials in which the within-S+ correlation was higher than the across class, was compared at each time point for data from anaesthetised mice (blue trace) and awake, behaving mice (black). See also Figure 5 - figure supplement 1. (D) SVM for discriminating EB vs. non-EB responses, trained on M/TC somatic responses from randomly selected $80 \%$ of trials, and tested on $20 \%$ of trials for anaesthetised (blue) and behaving (black) conditions. Both training data and test data contained random selection of single odour responses (hollow rectangles in scheme) and mixture responses (grey) rectangles. (E) SVM trained with single odour responses, tested on mixture responses. 
A

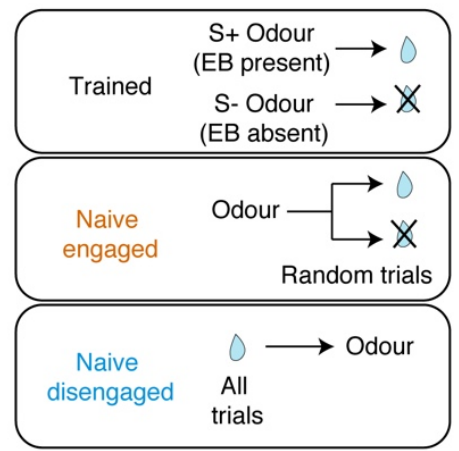

C
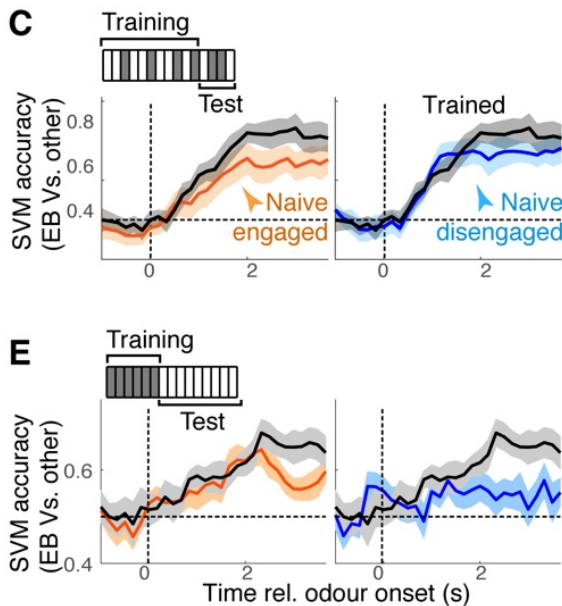

B Time to inh. peak

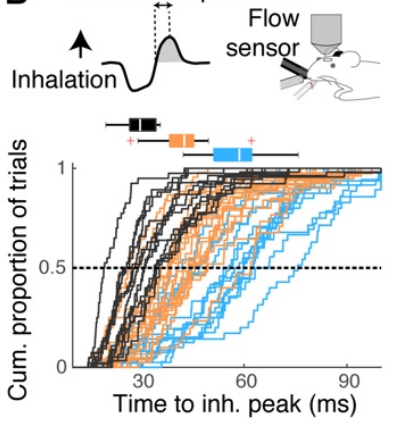

D
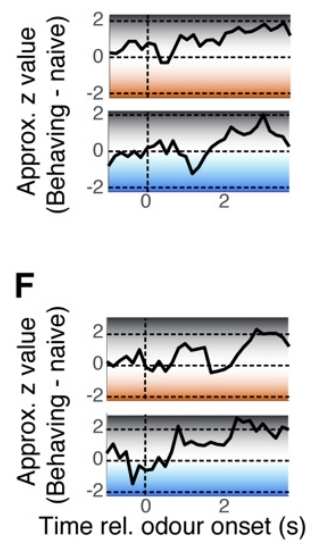

Figure 6: Mixture representation depends on behavioural states.

(A) Reward contingencies used to achieve different behavioural states in awake mice. Top: Mice were trained to discriminate between odours with EB vs. odours without EB. Middle: Naïve mice received water reward 3 seconds after the onset of odour, on randomly selected trials. Bottom: Naïve mice received water every trial, 15 seconds before odour onset. (B) Sniff patterns associated with trained and behaving mice (black), naïve and engaged mice (orange) and naïve and disengaged mice (light blue). Speed of inhalation during odour presentations shown. (C) Comparison of SVM performance using data from behaving mice (black) vs. naïve engaged mice (left panel, orange), and vs. naïve disengaged mice (right pane, light blue). SVM was trained to discriminate responses to S+ vs. S- odours using randomly selected $80 \%$ of trials and tested on the remaining $20 \%$ of the trials. (D) Decoder performance for the results in (C) plotted using approximate z-values obtained from Matlab implementation of Wilcoxon rank sum test. (E) As in (C), but SVM was trained using responses to single odours, and tested on mixture responses. (F) As in (D) but for the results in (E). $n=13$ fields of view, 6 mice for behaving, 17 fields of view, 6 mice for naive, engaged, and 14 sessions, 4 mice for naïve, disengaged mice. See Figure 6 - figure supplement 1 for linearity of summation. 


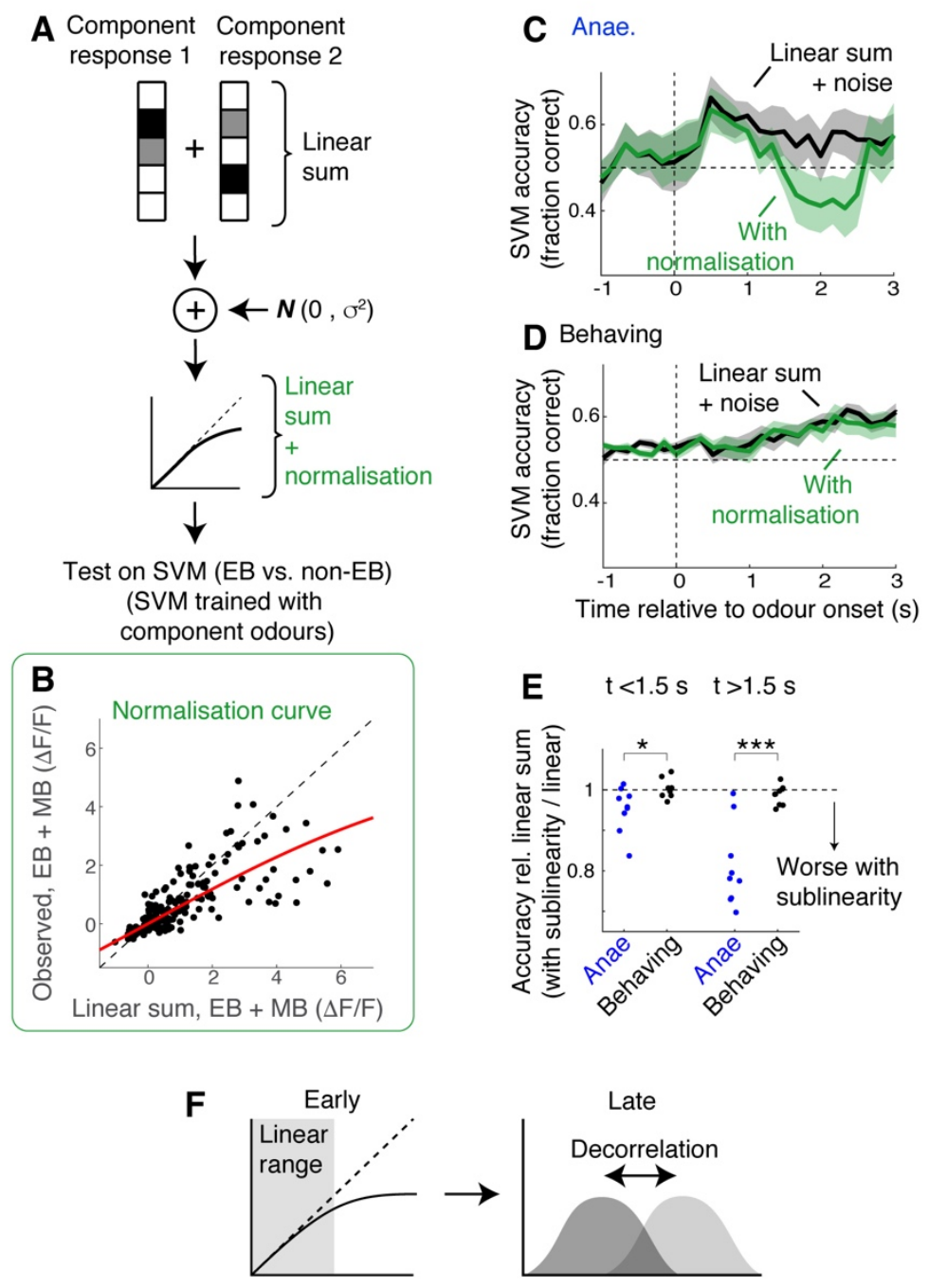

Figure 7: Simulation indicates data from behaving mice is less susceptible to normalizing sublinearity

(A) Simulation approach. For each field of view, component responses were averaged across trials and summed to obtain simulated mixture responses. Subsequently, uncorrelated Gaussian noise was added, and the summed responses were further transformed using a normalisation model (Mathis et al., 2016; Penker et al., 2020). These were then used as test inputs on SVMs that had been trained with single odour responses. (B) Parameter extraction. Scatter plot shows observed EB + MB mixture responses against linear sums of component responses (black dots). Red line shows the model fit used in the simulation. Data was from behaving mice, at 2 seconds after the odour onset. (C) Comparison of SVM performance using data from anaesthetised mice. Black line corresponds to linear sum + noise as inputs; Green line corresponds to the results with additional normalising sublinearity. Mean \pm SEM shown. (D) Same as in (C) but for data from awake, behaving mice. (E) Comparison of SVM performance. SVM accuracy for simulated mixture responses with sublinearity expressed as a fraction of accuracy obtained with simulated linear sum. $p=$ 0.03 for early phase $(0-1.5 \mathrm{~s})$ and $8.13^{*} 10^{-4}$ for late phase (1.5 $-3 \mathrm{~s}$ after odour onset); paired t-test. (F) Schematic of the finding: possible mechanisms of mixture representation in behaving mice may evolve over time. In awake, behaving mice, responses evoked in the $O B$ output neurons are dampened early on, remaining largely in the linear range of the normalization curve. Over time, the responses become larger, making sublinearity more widespread, but a pattern decorrelation may make evoked mixture responses 

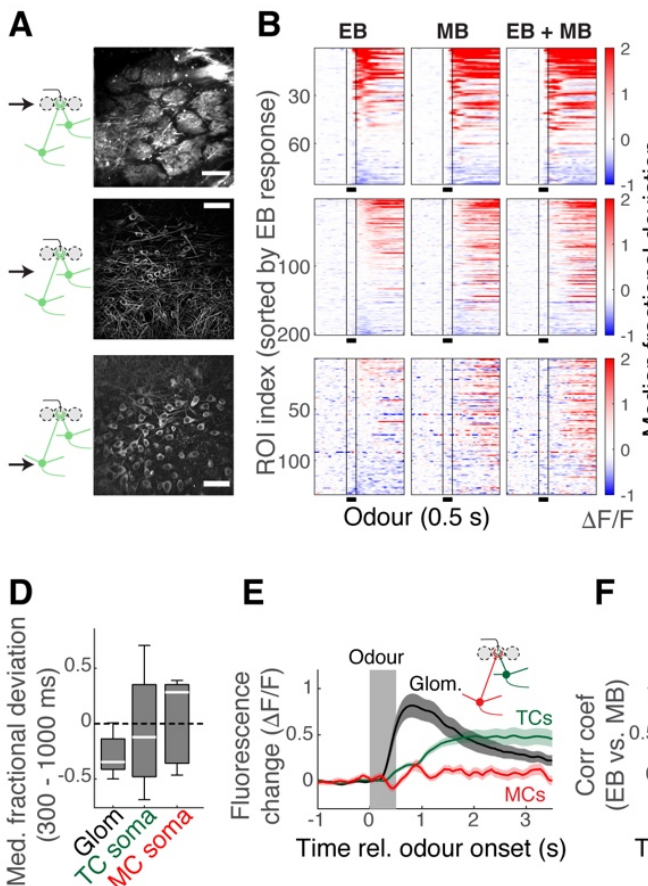

E

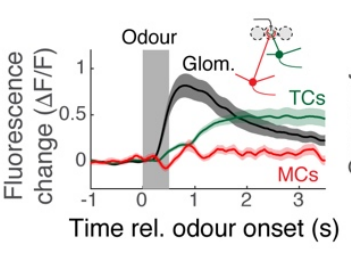

C

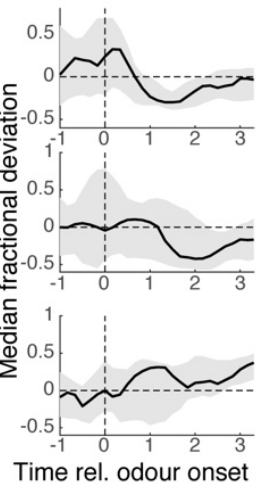

(s)

Figure 4 - figure supplement 1: Subcellular dependence on mixture summation properties

(A) Two-photon imaging of the OB output at three depths in Tbet-Cre::Ai95D mice performing the mixture task. Top: Imaging from the apical dendrites at the glomerular level. Middle: Imaging from the TC somata at the external plexiform layer. Bottom: Imaging from MC somata at the mitral cell layer. Scale bar $=50$ $\mu \mathrm{m}$. (B) Evoked responses in the apical dendrites (top), TC somata (middle), and MC somata (bottom) by ethyl butyrate on its own (left column), methyl butyrate on its own (middle column) and by the mixture of ethyl butyrate and methyl butyrate (right). (C) Median fractional deviation for all fields of view ( $n=7$ fields of view, 7 mice for apical dendrites; 13 fields of view, 6 mice for TC somata; 5 fields of view, 3 mice for MC somata). Thick lines correspond to the median, and shaded areas show the $25^{\text {th }}-75^{\text {th }}$ percentile range. (D) Boxplot summary of median fractional deviations for the imaged structures (not significant). (E) Average fluorescence change for all apical dendrites at the glomeruli (black trace), TC somata (green), and MC somata (red). Mean \pm s.e.m. shown. (F) Pearson's correlation coefficient between EB responses and MB responses from each field of view, averaged. Mean \pm SEM shown. 
bioRxiv preprint doi: https://doi.org/10.1101/2021.09.23.461425; this version posted February 22,2022 . The copyright holder for this preprint (which was not certified by peer review) is the author/funder, who has granted bioRxiv a license to display the preprint in perpetuity. It is made available under aCC-BY-ND 4.0 International license.

656

A

Early $(0-1 s) \quad$ Late $(2-3 s)$

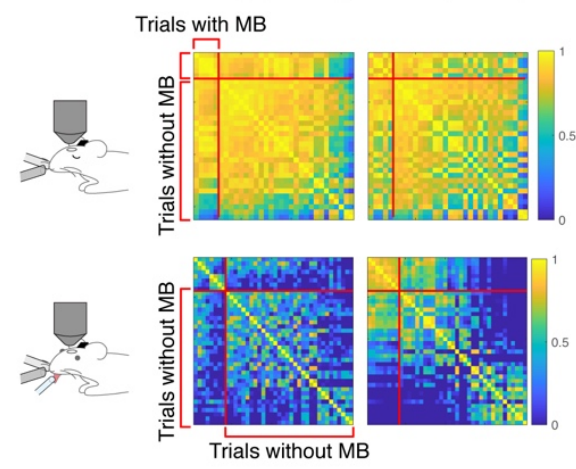

B

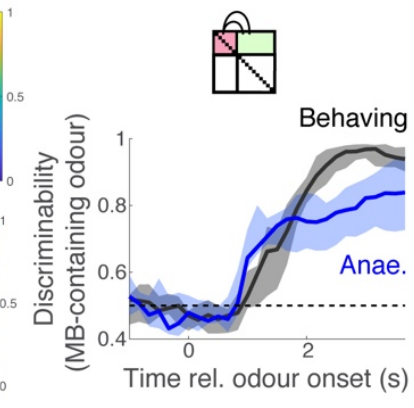

Figure 5 - figure supplement 1: Discriminability of $M B$ responses

(A) Correlation matrices were obtained in the same way as in Figure 5A, except that the trials are sorted by whether the stimuli contained methyl butyrate. (B) Discriminability index over time, where within-odour comparisons are between trials where methyl butyrate was presented. 
bioRxiv preprint doi: https://doi.org/10.1101/2021.09.23.461425; this version posted February 22,2022 . The copyright holder for this preprint (which was not certified by peer review) is the author/funder, who has granted bioRxiv a license to display the preprint in perpetuity. It is made available under aCC-BY-ND 4.0 International license.
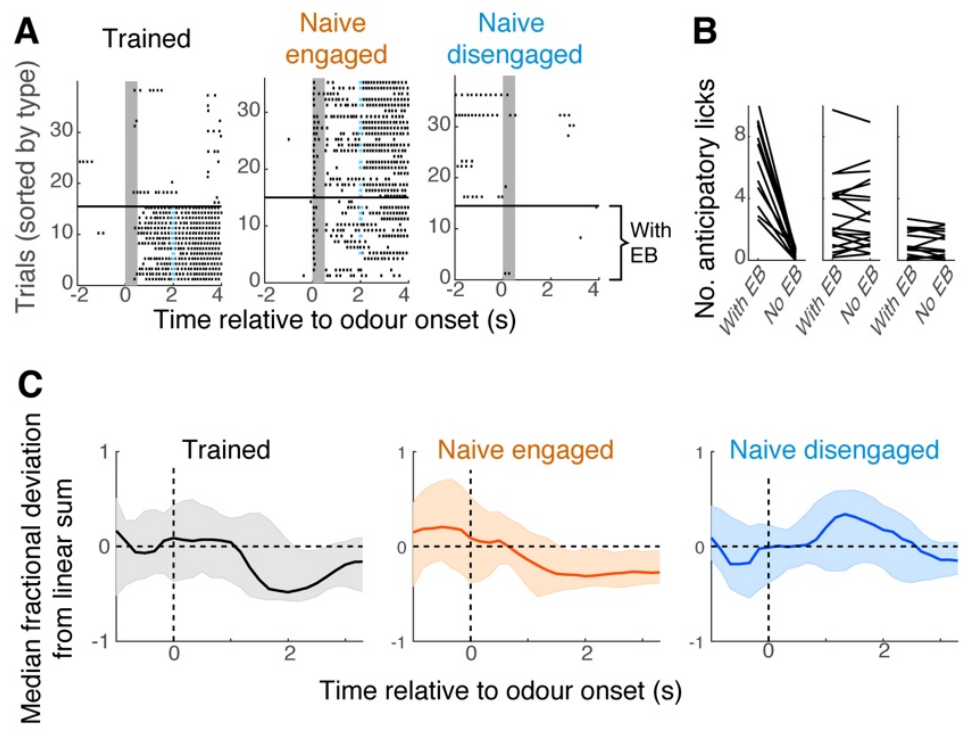

Figure 6 - figure supplement 1: Further characterization of awake behavioural states.

(A) Lick raster plots for example mice; left, trained mice performing the mixture task; middle, naïve engaged mice; right, naïve disengaged mice. Vertical grey bars indicate the time of odour presentation $(0.5$ s). Trials with ethyl butyrate presentations are ordered below the black horizontal bar. (B) Average number of anticipatory licks generated by mice in each group, on trials with EB vs. trials without EB. Each data point corresponds to each imaging session. (C) Time course of linearity in summation; median fractional deviation from each field of view is summarized here with median (thick line), and $25^{\text {th }}$ and $75^{\text {th }}$ percentile range (shaded areas). 
bioRxiv preprint doi: https://doi.org/10.1101/2021.09.23.461425; this version posted February 22,2022 . The copyright holder for this

preprint (which was not certified by peer review) is the author/funder, who has granted bioRxiv a license to display the preprint in perpetuity. It is made available under aCC-BY-ND 4.0 International license.

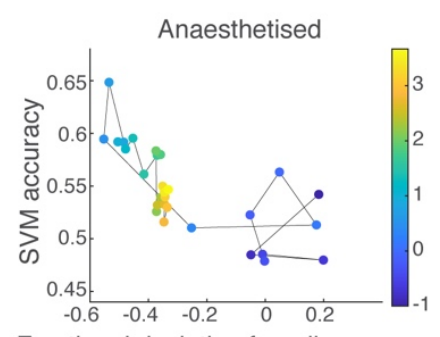

Fractional deviation from linear sum

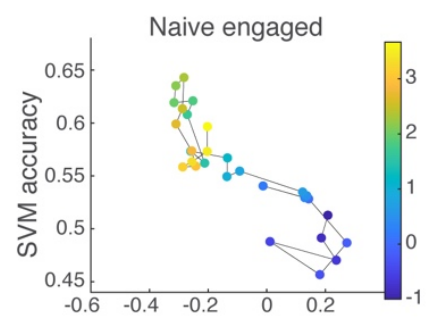

Fractional deviation from linear sum

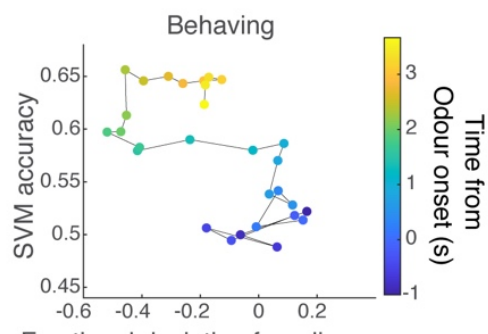

Fractional deviation from linear sum

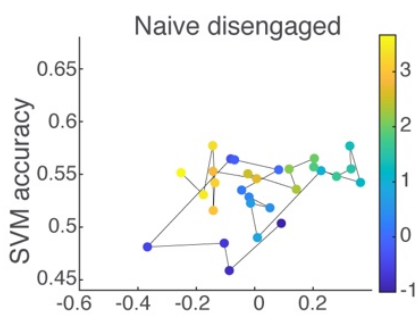

Fractional deviation from linear sum

Figure 6 - figure supplement 2: Relationship between discriminability and linearity of mixture summation.

Comparison of discriminability against the linearity of summation for all behavioural states. The average ability (fraction of trials correct) of SVMs (trained on single odour responses) to discriminate EB mixtures against non-EB mixtures is shown against the median fractional deviation from linear sum at the corresponding time. The time elapsed from the odour onset is colour coded. 
A
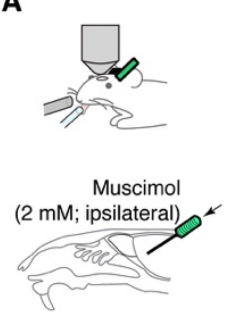

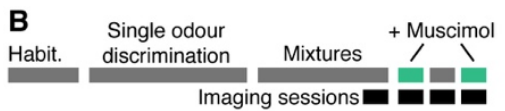

C
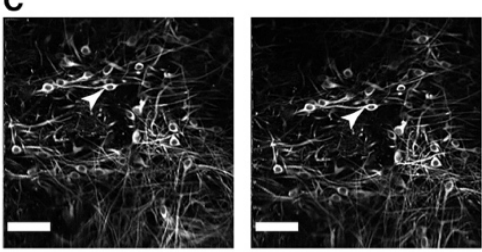

D
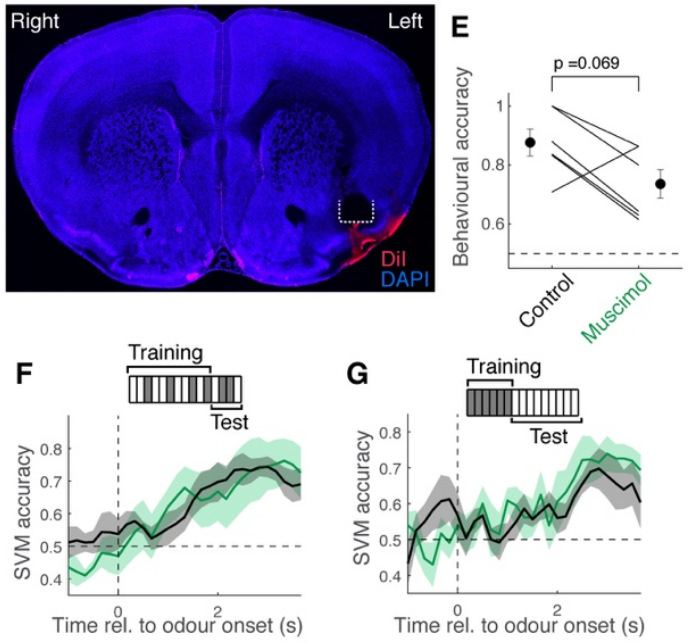

Figure 6 - figure supplement 2: Mixture representation in TCS is not affected by ipsilateral piriform inactivation.

(A) To assess if the learning-dependent improvement in the decoder performance requires an involvement of the piriform cortex, we infused muscimol ( $500 \mathrm{nl}, 2 \mathrm{mM}$ ) in the ipsilateral, anterior piriform cortex during imaging, while trained mice performed the mixture task. A cannula was implanted at an angle, in a manner similar to (Otazu et al., 2015) in order to infuse muscimol. (B) When the mice were trained to perform the task well, the imaging sessions commenced. Muscimol and control sessions alternated, where co-ordinates for the imaged fields of view were recorded and could be revisited, so that imaging could be obtained from the same location under the control and muscimol conditions. (C) Example matched fields of view for control session (left) and muscimol session (right). Arrow heads point to the same tufted cells imaged on different days. (D) Example confocal image of a coronal section of the brain, showing a track left by an implanted cannula (white dotted line) in the left piriform cortex. Blue = DAPI fluorescence; red = fluorescence from Dil, which was injected before perfusion. (E) Behavioural performance for control and muscimol sessions. Sessions with the common fields of view are paired. $P=0.069$, paired t-test $(n=6$ sessions, 3 mice). (F) SVM performance in the ability to discriminate S+ odours (EB present) vs. S- odours (EB absent), for control (black) and muscimol (green) datasets. Mean \pm s.e.m. shown. (G) Same as (F), except that the training set comprised responses from single odour trials, and the test set comprised mixture responses. 


\section{References}

Abraham, N.M., Egger, V., Shimshek, D.R., Renden, R., Fukunaga, I., Sprengel, R., Seeburg, P.H., Klugmann, M., Margrie, T.W., Schaefer, A.T., et al. (2010). Synaptic Inhibition in the Olfactory

Ackels, T., Erskine, A., Dasgupta, D., Marin, A.C., Warner, T.P.A., Tootoonian, S., Fukunaga, I., Harris, J.J., and Schaefer, A.T. (2021). Fast odour dynamics are encoded in the olfactory system and guide behaviour. Nature 593, 558-563.

721

Agmon-Snir, H., Carr, C.E., and Rinzel, J. (1998). The role of dendrites in auditory coincidence detection. Nature 393, 268-272.

Araneda, R.C., Kini, A.D., and Firestein, S. (2000). The molecular receptive range of an odorant receptor. Nature Neuroscience 3, 1248-1255.

Bell, G.A., Laing, D.G., and Panhuber, H. (1987). Odour mixture suppression: evidence for a peripheral mechanism in human and rat. Brain Research 426, 8-18.

Bhattacharjee, A.S., Konakamchi, S., Turaev, D., Vincis, R., Nunes, D., Dingankar, A.A., Spors, Reports 28, 2966-2978.e2965.

Bregman, A.S. (1990). Auditory Scene Analysis: The Perceptual Organization of Sound (The MIT Press).

Bridgeford, E.W., Wang, S., Wang, Z., Xu, T., Craddock, C., Dey, J., Kiar, G., Gray-Roncal, W., generalization error and maximize replicability: Applications in connectomics and genomics. PLOS Computational Biology 17, e1009279.

Buck, L., and Axel, R. (1991). A novel multigene family may encode odorant receptors: A molecular basis for odor recognition. Cell 65, 175-187.

Carey, R.M., and Wachowiak, M. (2011). Effect of Sniffing on the Temporal Structure of Mitral/Tufted Cell Output from the Olfactory Bulb. The Journal of Neuroscience 31, 10615.

Chae, H., Banerjee, A., and Albeanu, D.F. (2021). A non-canonical feedforward pathway for computing odor identity. bioRxiv, 2020.2009.2028.317248.

Chapuis, J., and Wilson, D.A. (2012). Bidirectional plasticity of cortical pattern recognition and behavioral sensory acuity. Nature Neuroscience 15, 155-161.

Cruz, G., and Lowe, G. (2013). Neural coding of binary mixtures in a structurally related odorant pair. Scientific Reports 3, 1220. 
Dana, H., Sun, Y., Mohar, B., Hulse, B.K., Kerlin, A.M., Hasseman, J.P., Tsegaye, G., Tsang, A., Wong, A., Patel, R., et al. (2019). High-performance calcium sensors for imaging activity in neuronal populations and microcompartments. Nature Methods 16, 649-657.

del Mármol, J., Yedlin, M.A., and Ruta, V. (2021). The structural basis of odorant recognition in insect olfactory receptors. Nature 597, 126-131.

Economo, M.N., Hansen, K.R., and Wachowiak, M. (2016). Control of Mitral/Tufted Cell Output by Selective Inhibition among Olfactory Bulb Glomeruli. Neuron 91, 397-411.

Firestein, S., Picco, C., and Menini, A. (1993). The relation between stimulus and response in olfactory receptor cells of the tiger salamander. The Journal of Physiology 468, 1-10.

Fletcher, M.L. (2011). Analytical Processing of Binary Mixture Information by Olfactory Bulb Glomeruli. PLOS ONE 6, e29360.

Friedrich, R.W., and Wiechert, M.T. (2014). Neuronal circuits and computations: Pattern decorrelation in the olfactory bulb. FEBS Letters 588, 2504-2513.

Fukunaga, I., Berning, M., Kollo, M., Schmaltz, A., and Schaefer, Andreas T. (2012). Two Distinct Channels of Olfactory Bulb Output. Neuron 75, 320-329.

Grabska-Barwińska, A., Barthelmé, S., Beck, J., Mainen, Z.F., Pouget, A., and Latham, P.E. (2017). A probabilistic approach to demixing odors. Nature Neuroscience 20, 98-106.

Gschwend, O., Abraham, N.M., Lagier, S., Begnaud, F., Rodriguez, I., and Carleton, A. (2015). Neuronal pattern separation in the olfactory bulb improves odor discrimination learning. Nature Neuroscience 18, 1474-1482.

Gupta, P., Albeanu, D.F., and Bhalla, U.S. (2015). Olfactory bulb coding of odors, mixtures and sniffs is a linear sum of odor time profiles. Nature Neuroscience 18, 272-281.

Haddad, R., Lanjuin, A., Madisen, L., Zeng, H., Murthy, V.N., and Uchida, N. (2013). Olfactory cortical neurons read out a relative time code in the olfactory bulb. Nature Neuroscience 16, 949.

Hopfield, J.J. (1991). Olfactory computation and object perception. Proceedings of the National Academy of Sciences 88, 6462.

Ilc, T., Werck-Reichhart, D., and Navrot, N. (2016). Meta-Analysis of the Core Aroma Components of Grape and Wine Aroma. Frontiers in Plant Science 7, 1472.

Inagaki, S., Iwata, R., Iwamoto, M., and Imai, T. (2020). Widespread Inhibition, Antagonism, and Synergy in Mouse Olfactory Sensory Neurons In Vivo. Cell Reports 31, 107814. 
Jacob, V., Le Cam, J., Ego-Stengel, V., and Shulz, D.E. (2008). Emergent Properties of Tactile Scenes Selectively Activate Barrel Cortex Neurons. Neuron 60, 1112-1125.

Jinks, A., and Laing, D.G. (1999). A Limit in the Processing of Components in Odour Mixtures. Perception 28, 395-404.

Jordan, R., Fukunaga, I., Kollo, M., and Schaefer, A.T. (2018). Active Sampling State Dynamically Enhances Olfactory Bulb Odor Representation. Neuron 98, 1214-1228.e1215.

Kay, L.M., Lowry, C.A., and Jacobs, H.A. (2003). Receptor contributions to configural and elemental odor mixture perception. Behavioral Neuroscience 117, 1108-1114.

Koldaeva, A., Schaefer, A.T., and Fukunaga, I. (2019). Rapid task-dependent tuning of the mouse olfactory bulb. eLife 8 , e43558.

Kurahashi, T., Lowe, G., and Gold, G.H. (1994). Suppression of odorant responses by odorants in olfactory receptor cells. Science $265,118$.

Laing, D.G., and Francis, G.W. (1989). The capacity of humans to identify odors in mixtures. Physiology \& Behavior 46, 809-814.

Laing, D.G., and Glemarec, A. (1992). Selective attention and the perceptual analysis of odor mixtures. Physiology \& Behavior 52, 1047-1053.

Laughlin, S.B. (1989). The role of sensory adaptation in the retina. Journal of Experimental Biology 146, 39-62.

Madisen, L., Garner, Aleena R., Shimaoka, D., Chuong, Amy S., Klapoetke, Nathan C., Li, L., van der Bourg, A., Niino, Y., Egolf, L., Monetti, C., et al. (2015). Transgenic Mice for Intersectional Targeting of Neural Sensors and Effectors with High Specificity and Performance. Neuron 85, 942-958.

Malnic, B., Hirono, J., Sato, T., and Buck, L.B. (1999). Combinatorial Receptor Codes for Odors. Cell 96, 713-723.

Marr, D. (1982). Vision: A Computational Investigation into the Human Representation and Processing of Visual Information (W.H.Freeman and Company).

Mathis, A., Rokni, D., Kapoor, V., Bethge, M., and Murthy, V.N. (2016). Reading Out Olfactory Receptors: Feedforward Circuits Detect Odors in Mixtures without Demixing. Neuron 91, 1110-1123.

Oka, Y., Omura M Fau - Kataoka, H., Kataoka H Fau - Touhara, K., and Touhara, K. Olfactory receptor antagonism between odorants.

Otazu, Gonzalo H., Chae, H., Davis, Martin B., and Albeanu, Dinu F. (2015). Cortical Feedback Decorrelates Olfactory Bulb Output in Awake Mice. Neuron 86, 1461-1477. 
850

851

852

853

854

855

856

857

858

859

860

861

862

863

864

865

866

867

868

869

870

871

872

873

874

875

876

877

878

879

880

881

882

883

884

885

886

887

888

889

890

891

892

893

894

895

Padmanabhan, K., and Urban, N.N. (2010). Intrinsic biophysical diversity decorrelates neuronal firing while increasing information content. Nature Neuroscience 13, 1276-1282.

Penker, S., Licht, T., Hofer, K.T., and Rokni, D. (2020). Mixture Coding and Segmentation in the Anterior Piriform Cortex. Frontiers in Systems Neuroscience 14, 89.

Poupon, D., Fernandez, P., Boisvert, S.A., Migneault-Bouchard, C., and Frasnelli, J. (2018). Can the identification of odorants within a mixture be trained? Chemical Senses 43, 721-726.

Reddy, G., Zak, J.D., Vergassola, M., and Murthy, V.N. (2018). Antagonism in olfactory receptor neurons and its implications for the perception of odor mixtures. eLife 7, e34958.

Rinberg, D., Koulakov, A., and Gelperin, A. (2006). Speed-Accuracy Tradeoff in Olfaction. Neuron 51, 351-358.

Rokni, D., Hemmelder, V., Kapoor, V., and Murthy, V.N. (2014). An olfactory cocktail party: figure-ground segregation of odorants in rodents. Nature Neuroscience 17, 1225-1232.

Rokni, D., and Murthy, V.N. (2014). Analysis and Synthesis in Olfaction. ACS Chemical Neuroscience 5, 870-872.

Singh, V., Murphy, N.R., Balasubramanian, V., and Mainland, J.D. (2019). Competitive binding predicts nonlinear responses of olfactory receptors to complex mixtures. Proceedings of the National Academy of Sciences 116, 9598.

Stettler, D.D., and Axel, R. (2009). Representations of Odor in the Piriform Cortex. Neuron 63, 854-864.

Tabor, R., Yaksi, E., Weislogel, J.-M., and Friedrich, R.W. (2004). Processing of Odor Mixtures in the Zebrafish Olfactory Bulb. The Journal of Neuroscience 24, 6611.

Verhagen, J.V., Wesson, D.W., Netoff, T.I., White, J.A., and Wachowiak, M. (2007). Sniffing controls an adaptive filter of sensory input to the olfactory bulb. Nature Neuroscience 10, 631-639.

Wachowiak, M., and Cohen, L.B. (2001). Representation of Odorants by Receptor Neuron Input to the Mouse Olfactory Bulb. Neuron 32, 723-735.

Weiss, T., Snitz, K., Yablonka, A., Khan, R.M., Gafsou, D., Schneidman, E., and Sobel, N. (2012). Perceptual convergence of multi-component mixtures in olfaction implies an olfactory white. Proceedings of the National Academy of Sciences 109, 19959.

Wilson, C.D., Serrano, G.O., Koulakov, A.A., and Rinberg, D. (2017). A primacy code for odor identity. Nature Communications 8, 1477. 
896 Wilson, D.A., and Stevenson, R.J. (2003). The fundamental role of memory in olfactory perception. Trends in Neurosciences 26, 243-247.

899 Xu, L., Li, W., Voleti, V., Zou, D.-J., Hillman, E.M.C., and Firestein, S. (2020). Widespread 900 receptor-driven modulation in peripheral olfactory coding. Science 368, eaaz5390.

Zak, J.D., Reddy, G., Vergassola, M., and Murthy, V.N. (2020). Antagonistic odor interactions in olfactory sensory neurons are widespread in freely breathing mice. Nature 904 Communications 11, 3350.

905

906 